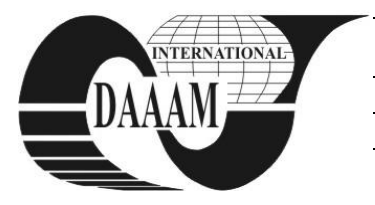

COMPARISON RATE WAVELET TRANSFORM FOR SELECTED TYPES

\title{
JURIK, M[ilan]
}

\begin{abstract}
The following article describes the wavelet transform comparing the selected types with regard to its speed. The four types selected include two types of the continuous and two types of the discrete wavelet transform. The transforms were programmed and then compared in the Wolfram Mathematica computing environment.
\end{abstract}

Key words: wavelet transform, transform, Haar, Daubechies

\section{INTRODUCTION}

To analyse signals, it is often convenient to use a transform which presents a signal in other than time (positional) domain. The wavelet transform decomposes a signal into a set of basic functions. These functions are the basis of a wavelet. The wavelets are obtained from the basic prototype $y(t)$, called mother wavelet, according to dilation and arrangement. In the transform, it is necessary to change the scale in the horizontal axis (frequency), vertical axis (amplitude) and shift the wavelet along the horizontal axis (position). This results in coefficients which determine the signal. These parameters are termed as scaling function $\Phi(\mathrm{x})$. The shape of the wavelet is determined by the wavelet function $\psi(\mathrm{x})$.

The main idea of the wavelet transform (WT) is to achieve an optimum ratio of resolution in time and frequency by changing a window width in time and by its suitable shape. The window is wider for low frequencies and narrower for high frequencies. This window is called mother wavelet $\psi$.

The input signal passes through two filters, a high pass filter $\mathrm{g}[\mathrm{n}]$ and a low pass filter $\mathrm{h}[\mathrm{n}]$. The wavelet function $\psi$ behaves as a high pass filter filtering the input signal from the middle of the frequency band towards higher frequencies. In the selected scale however, the band from lower frequencies to zero remains uncovered. Scaling function $\Phi$ of low pass filter character is derived from the wavelet function $\psi$. Filters $g[n]$ and $h[n]$ form a pair known as a quadrature mirror filter (QMF) (Percival \& Walden, 2000).

The outputs from both of the filters are down sampled to the half of the input samples (every second signal value is taken). The high pass filter provides us so called DWT detail coefficients while approximation coefficients are provided by the low pass filter. Therefore, because of down sampling, the total number of coefficients after the first step is the same as the number of the input samples. The approximation coefficients can further be analyzed by the same decomposition to obtain a further set of approximation coefficients and details. This procedure can be repeated on and on until we run out of the samples.

\section{DIGITAL FILTERS}

A filter is seen as a time-independent operator which influences the system frequency characteristics and can be expressed as follows:

$$
y(n)=\sum_{k} h(k) * x(n-k)
$$

where $x$ is a vector of the input signal, $y$ is a vector of the output signal and $h$ is a vector of the coefficients characterizing filter transfer characteristics.

A low pass filter is used to analyze low frequency signals and it can be expressed by the relation:

$$
y_{L P}(n)=\frac{1}{2} x(n)+\frac{1}{2} x(n-1)
$$

This filter type is also called the moving average and it smoothes out rapid changes in the signal.

A high pass filter is used to analyze higher frequency signals and it can be expressed by the relation:

$$
y_{H P}(n)=\frac{1}{2} x(n)-\frac{1}{2} x(n-1)
$$

This filter type is also called the moving difference and it captures rapid changes in the signal (Weeks, 2007).

\section{CWT AND DWT}

Defining relation for the CWT (continuous wavelet transform) can be defined as:

$$
W_{(s, \tau)}=\int f(t) \psi_{s, \tau}^{*}(t) d t
$$

where $*$ is a complex variable. This relation represents decomposition of the function $f(t)$ into a set of basic functions $\psi s \tau(\mathrm{t})$ called wavelets here.

The wavelets are generated from a single basis function $\psi(t)$, so called mother wavelet.

$$
\psi_{s, \tau}(t)=\frac{1}{\sqrt{|s|}} \psi\left(\frac{t-\tau}{s}\right)
$$

Using the parameter $S$, called scale, it is possible to measure the wavelet width (dilation). Parameter $\tau$, called position, changes the position of the wavelet on the time axis (translation).

Due to several properties, it is extremely difficult to apply the continuous wavelet transform in practice. Looking at the equation describing the CWT, it is clear that the CWT computation is largely redundant as the wavelet is continuously shifted along the signal being analyzed while the scaling occurs continuously as well. Therefore, the transform output will be an infinite number of coefficients which are redundant for practical application.

The discrete wavelet is then:

$$
\psi_{p, k}(t)=\frac{1}{\sqrt{\left|2^{p}\right|}} \psi\left(\frac{t-2^{p} * k}{2^{p}}\right)
$$


Parameter $P$ corresponds to the scale, $k$ position (Bachman et al., 2000).

\section{TRANSFORM TYPES}

A notebook with software Mathematica, version 8 by Wolfram was employed to create algorithms and their subsequent graphic visualization and comparison. Hardware:

- Intel Core 2 duo $2.0 \mathrm{GHz}$

- Memory: 2 GB RAM

- Graphics card: ATI RADEON X2300

Two types of mother wavelets from the CWT (continuous wavelet transform) and two types from the DWT (discrete wavelet transform) were used in the following visualization and comparison.

\subsection{Types of the discrete wavelet transform}

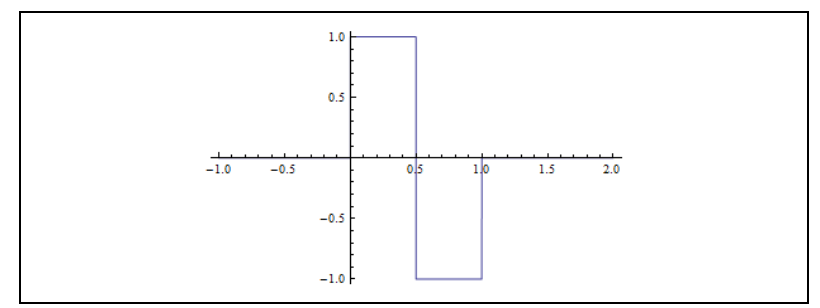

Fig. 1. Haar wavelet

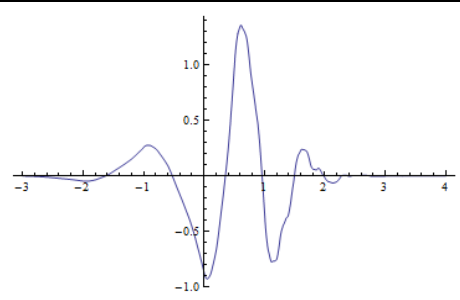

Fig. 2. Daubechies wavelet

\subsection{Types of the continuous wavelet transform}

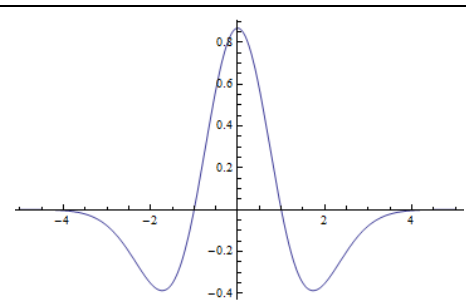

Fig. 3. Mexican Hat wavelet

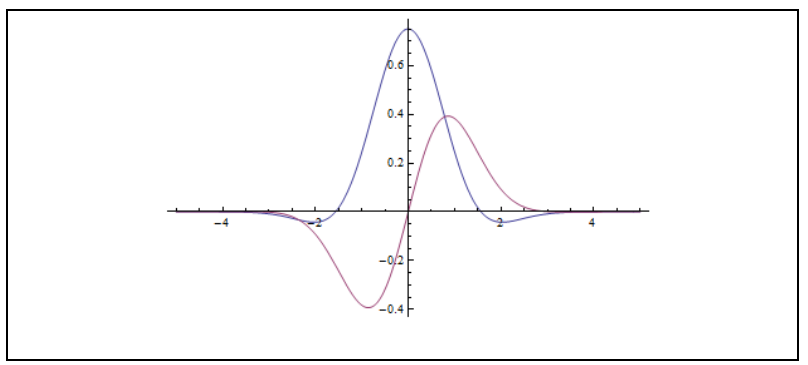

Fig. 4. Gabor wavelet

\section{COMPARISON RESULTS}

In the measurement, I proceeded by choosing the following five numerical value arrays: 128, 512, 1024, 2048 and 4096.
Each array had a different speed of processing, i.e. each transform achieved a different length of the processing time.

\begin{tabular}{|c|c|c|c|c|}
\hline \multirow{2}{*}{$\begin{array}{c}\text { Number } \\
\text { of values }\end{array}$} & \multicolumn{4}{|c|}{ Transform types } \\
\cline { 2 - 5 } & Haar & Daubechies & Mexican & Gabor \\
\hline 128 & $0.005 \mathrm{~s}$ & $0.008 \mathrm{~s}$ & $0.015 \mathrm{~s}$ & $0.016 \mathrm{~s}$ \\
\hline 512 & $0.006 \mathrm{~s}$ & $0.009 \mathrm{~s}$ & $0.017 \mathrm{~s}$ & $0.018 \mathrm{~s}$ \\
\hline 1024 & $0.008 \mathrm{~s}$ & $0.011 \mathrm{~s}$ & $0.019 \mathrm{~s}$ & $0.02 \mathrm{~s}$ \\
\hline 2048 & $0.015 \mathrm{~s}$ & $0.016 \mathrm{~s}$ & $0.031 \mathrm{~s}$ & $0.031 \mathrm{~s}$ \\
\hline 4096 & $0.02 \mathrm{~s}$ & $0.022 \mathrm{~s}$ & $0.047 \mathrm{~s}$ & $0.048 \mathrm{~s}$ \\
\hline
\end{tabular}

Tab 1. Obtained values for each transform

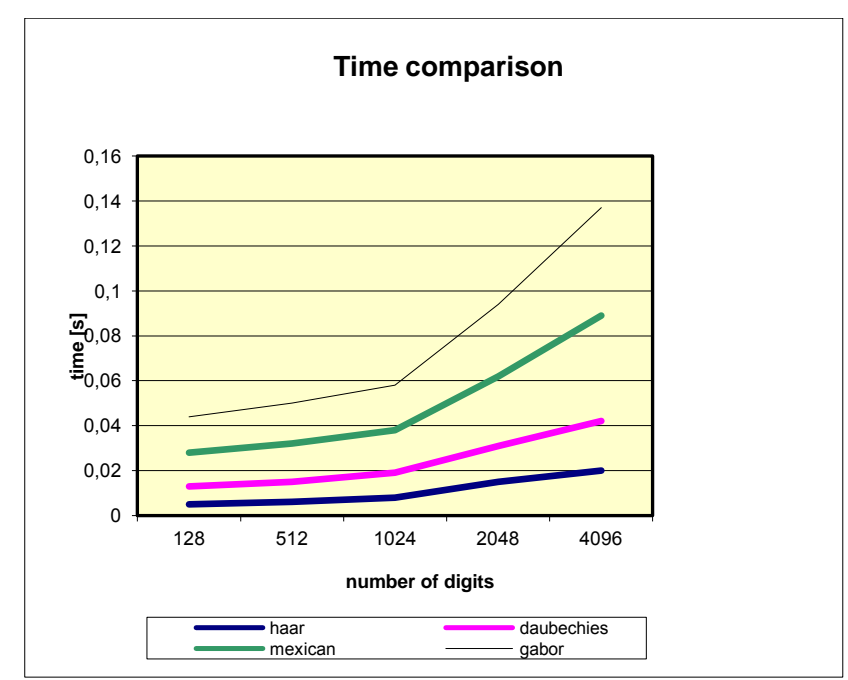

Fig. 5. Dependence of the transform speed on the number of values

\section{CONCLUSION}

In this article, the wavelet transform together with its four types is described, followed by its division into the continuous and discrete transform. Using the Wolfram Mathematica 8 environment, the aim of the article was to program four types of transforms and compare them with regard to the speed of processing of the given value array. The results show that both of the discrete transforms, Haar and Daubechies, are significantly faster than the continuous transforms, Mexican and Gabor. The measured values were under 1 second, therefore the processing time of the value array would linearly grow with its size.

\section{ACKNOWLEDGEMENTS}

This work was supported by the Internal Grant Agency of Tomas Bata University in Zlin, project no. IGA/49/FAI/10/D.

\section{REFERENCES}

Donald B. Percival, Andrew T. Walden (2000), Wavelet Methods for Time Series Analysis, Cambridge University Press, ISBN: 978-0-521-68508-5 paperback

Michael Weeks (2007), Digital Signal Processing Using Matlab and Wavelets, Infinity Science Press LLC, ISBN: 09778582-0-0

George Bachman, Lawrence Narici, Edward Beckenstein (2000), Fourier and Wavelet Analysis, Springer-Verlag New York, Inc. ISBN: 0-387-98899-8

Yves Nievergelt (2001), Wavelets made easy, Birkhäuser Boston, ISBN: 3-7643-4061-4

Gerald Kaiser (2010), A Friendly Guide to Wavelets, Birkhäuser, ISBN: 978-0-8176-8110 